\title{
Doxorubicin resistance induces IL6 activation in the colon cancer cell line LS180
}

\author{
XIAO-YUN LI* ${ }^{*}$ XIAO-FENG LIAO* ${ }^{*}$ HONG-BO WANG and JIAN ZHANG \\ Department of General Surgery, Xiangyang Central Hospital, Affiliated Hospital of Hubei College of Arts and Science, \\ Xiangyang, Hubei 441021, P.R. China
}

Received September 22, 2017; Accepted July 23, 2018

DOI: $10.3892 / \mathrm{ol} .2018 .9360$

\begin{abstract}
Despite improvements in the development of drugs for the treatment of cancer, drug resistance remains a major obstacle. In colon cancer, following an initially promising response, , patients develop drug resistance, which impacts the efficacy and halts the response of cancerous cells towards drugs. In the present study, a phosphatase and tensin homolog (PTEN) knockdown model of LS180 cells, doxorubicin-resistant models of LS180 cells as well as doxorubicin-resistant LS180 (PTEN) knockdown model were established. The present study demonstrated that doxorubicin resistance led to the activation of interleukin (IL)6 signalling pathway which was enhanced by knockdown of PTEN. There was also an increase in the levels of IL8 and IL2 which were further enchanced by knockdown of PTEN. Doxorubicin resistance also led to an increase in the population of cancer stem cells in LS180 and shPTEN-treated LS180 cells. Notably, doxorubicin resistance also induced epithelial to mesenchymal transition and increased the formation of mammospheres. Furthermore, the present study also reported that IL6 receptor antibody not only decreased IL6 levels but also led to a significant decreased number of cancer stem cell like population and mammosphere formation. In conclusion, in the present study it was demonstrated that doxorubicin resistance led to activation of IL6 signalling pathway which was further elevated by the knockdown of PTEN in the colon cancer cell line LS180. Thus, inhibiting the IL6 loop may provide an alternative pathway to tackle doxorubicin resistance.
\end{abstract}

Correspondence to: Mr. Jian Zhang, Department of General Surgery, Xiangyang Central Hospital, Affiliated Hospital of Hubei College of Arts and Science, 39 Jingzhou Street, Xiangyang, Hubei 441021, P.R. China

E-mail: ethastoklonso@yahoo.com

*Contributed equally

Key words: colon cancer, doxorubicin, resistance, cancer stem cells, epithelial-mesenchymal transition

\section{Introduction}

Among various types of cancer, colon cancer is one of the leading causes of cancer-associated mortalities worldwide (1). Colon cancer involves the formation of malignant tumors in the colon tissues and is one of the most commonly diagnosed types of cancer (2). Currently, patients with colon cancer undergo two main treatment options i.e., chemotherapy and surgery, and among the two medical procedures the one that is used depends upon the size of the tumor and stage of cancer in patients $(3,4)$. Whereas in a protion of patients surgery is followed by chemotherapy, there are other patients to whom chemotherapy is initially administered to reduce the size of the tumor, which is then typically followed by surgery (5-7). However despite the advances made in medical sciences over the last decade, drug resistance remains a principal reason for treatment failure (8-10). In colon cancer, patients develop drug resistance with the passage of treatment and ultimately stop responding to the available treatment options, which culminates in the failure of chemotherapy $(11,12)$. Drug resistance is defined as a decrease in the effect of drugs, including chemotherapeutic agents or antibiotics, and targeting drug resistance, primarily multidrug resistance, remains one a major challenge $(13,14)$.

Phosphatase and tensin homolog (PTEN) a tumor suppressor gene, has been reported to be involved in various types of cancer $(15,16)$. Mutations in PTEN have been reported to be involved in the development of cancer $(17,18)$. In various types of cancer the mutation frequency of PTEN is very high (19). PTEN (phosphatase and tensin homologue) is a phosphatase that dephosphorylates both protein and phosphoinositide substrates that regulates longevity $(20,21)$. The intracellular levels of phosphatidylinositol are negatively regulated by PTEN which acts as a tumor suppressor by negatively regulating the Akt signaling pathway; a pathway that has been demonstrated to be deregualted in the majority of cancers (22). Mutations in this gene contribute to the failure of chemotherapy and therefore drug resistance (23).

Overproduction of interleukin (IL) 6 and 8 may be caused due to decreased expression of PTEN as well as drug resistance, and it has been hypothesized to be involved in the expansion of cancer stem cell population in tumors (24). A positive feedback loop is generated whereby IL6 activates the nuclear factor $(\mathrm{NF})-\kappa \mathrm{B}$ signalling pathway that 
further enhances the production of IL6 creating a positive feedback loop, thus linking inflammation to the malignant transformation of tumors (25). It has also been reported that the levels of IL6 in patients directly correlate with their overall survival rate (26). Therefore, studying drug resistance and the underlying mechanisms is of great clinical importance.

\section{Materials and methods}

Drugs, reagents and chemicals. Doxorubicin was purchased from Selleck Chemicals (Houston, TX, USA). RPMI-1640, radioimmunoprecipitation assay (RIPA) buffer, Hanks buffer and MTT reagent were obtained from Sigma-Aldrich (Merck KGaA; Darmstadt, Germany). Tocilizumab (an anti-IL6R antibody) was purchased from Roche Diagnostics, Basel, Switzerland. Primers, probes and cDNA kits for mRNA quantification were obtained from Invitrogen (Thermo Fisher Scientific, Inc., Waltham, MA, USA) and foetal bovine serum (FBS), Lipofactamine 2000 and Antibiotic-Antimycotic were procured from Gibco (Thermo Fisher Scientific, Inc). Lentiviral particles for knockdown of PTEN were obtained from Santa Cruz Biotechnology, Inc. (Dallas, TX, USA). All antibodies were obtained from CST (Cell Signaling Technology, Inc., Danvers, MA, USA).

Cell culture conditions. The LS180 cell line was obtained from American Type Culture Collection (Manassas, VA, USA) and grown in RPMI-1640 supplemented with $1 \%$ antibiotics Antibiotic-Antimycotic and $10 \% \mathrm{FBS}$ at $37^{\circ} \mathrm{C}$ in a humidified atmosphere containing $5 \% \mathrm{CO}_{2}$. For the generation of doxorubicin-resistant cells, LS180 and LS180 short hairpin (sh)PTEN (shPTEN-treated LS180) cells were treated with $1 \mu \mathrm{M}$ of doxorubicin for a period of nine months. However, resistant cells were grown in drug-free media for $48 \mathrm{~h}$ prior to experimentation.

PTEN knockdown. shRNA PTEN lentiviral particles from Santa Cruz Biotechnology, Inc. (Dallas, TX, USA) were used for PTEN knockdown. Briefly,LS180 and doxorubicin-resistant LS180 cells were grown in 6 well plates in an incubator at $37^{\circ} \mathrm{C}$ with $5 \% \mathrm{CO}_{2}$ and $95 \%$ humidity for $24 \mathrm{~h}$. shRNA PTEN Lentiviral particles Santa Cruz Biotechnology Inc. at a concentration of $1 \mu \mathrm{g} / \mathrm{ml}$ with polybrene [Sigma-Aldrich $(5 \mu \mathrm{g} / \mathrm{ml})]$ were transfected using Lipofectamine 2000 (Invitrogen; Thermo Fisher Scientific, Inc.) to the cells for $24 \mathrm{~h}$. Following this $24 \mathrm{~h}$ incubation, media was replaced with complete DMEM. Puromycin $(4 \mu \mathrm{g} / \mathrm{ml})$ was used as a selection marker and three rounds of selection were performed for $48 \mathrm{~h}$ each. Only the cells resistant to puromycin were cultured for subsequent experiments.

Cell proliferation assay. The LS180 cell line and its LS180 PTEN knockdown model, as well as doxorubicin-resistant cells were seeded in 96-well plates at a density of $1.5 \times 10^{4}$ cells $/$ well and allowed to grow for $24 \mathrm{~h}$ at $37^{\circ} \mathrm{C}$. At $24 \mathrm{~h}$, parental and resistant cells were treated with doxorubicin at a concentration dependent manner $(0.1,0.2$, $0.4,0.8,1,2,5,10,15$ and $20 \mu \mathrm{M}$ ) for $48 \mathrm{~h}$. MTT solution was added into each well at a concentration of $2.5 \mathrm{mg} / \mathrm{ml}$ and cells were incubated for $4 \mathrm{~h}$ at $37^{\circ} \mathrm{C}$. Finally, a total of
$150 \mu \mathrm{l}$ dimethylsulfoxide (DMSO) was added to each well to dissolve the formazen crystals and absorbance was detected at $570 \mathrm{~nm}$ by a synergy MX plate reader (BioTek Instruments, Inc., Winooski, VT, USA).

Western blotting. LS180, LS180 shPTEN, doxorubicin LS180 and doxorubicin LS180 shPTEN cells were seeded at a density of $1 \times 10^{6}$ in $60 \mathrm{~mm}$ dishes for $24 \mathrm{~h}$. Cells were treated with doxorubicin at a concentration of $1 \mu \mathrm{M}$ for $24 \mathrm{~h}$ and were lysed using RIPA buffer. Protein determination was performed by the Bradford method, and proteins $(70 \mu \mathrm{g})$ were separated on $10 \%$ SDS-PAGE and then transferred onto a nitrocellulose membrane at $100 \mathrm{~V}$ for $2 \mathrm{~h}$. To avoid non-specific binding, membranes were blocked in 5\% fat-free milk for $1 \mathrm{~h}$ at room temperature and primary antibodies p-AKT ser473 (10\% SDS-PAGE; cat. no. 4060; dilution 1:1,000), AKT (10\% SDS-PAGE; cat. no. 4691; dilution 1:1,000), NF-кB p65 (10\% SDS-PAGE; cat. no. 8242; dilution 1:1,000), EpCAM (10\% SDS-PAGE; cat. no. 93790; dilution 1:1,000), claudin-3; 15\% SDS-PAGE; cat. no. 83609; dilution 1:1,000), PTEN; 10\% SDS-PAGE; cat. no. 9188; dilution 1:1,000), TGFR2 (10\% SDS-PAGE; cat. no. 79424; dilution 1:1,000), vimentin (10\% SDS-PAGE; cat. no. 5741; dilution 1:1,000), $\beta$-actin (cat. no. 4970; dilution 1:2,000), TWIST (15\% SDS-PAGE; cat. no. 14472; dilution 1:1,000), E-cadherin (6\% SDS-PAGE; cat. no. 14472; dilution 1:1,000), HRP conjugated mouse anti-rabbit secondary antibody (cat. no. 93702; dilution 1:2,500), anti-mouse secondary antibody (cat. no. 14709; dilution 1:2,500) were obtained from Cell Signaling Technology Inc., (Danvers, MA, USA) incubated overnight at $4^{\circ} \mathrm{C}$. Membranes were washed with Tris-buffered saline containing $0.05 \%$ Tween-20 (TBST) two times for $5 \mathrm{~min}$. The horseradish peroxidase (HRP)-labeled antibody was added at room temperature for $1 \mathrm{~h}$, after which the protein blots were again washed twice with TBST at room temperature for $5 \mathrm{~min}$ each. Finally, protein bands were visualized using enhanced chemiluminescence (ECL; GE Healthcare, Chicago, IL, USA) and an X-ray film.

CD44/CD24 assay. This assay was performed to detect the cancer stem cell fraction within the colon cancer cell line LS180. Cells (LS180, LS180 shPTEN, doxoLS180 and doxoLS180 shPTEN) were incubated with anti-CD44-PE (cat. no. ab46793; dilution 1:200) and anti-CD24-FITC (cat. no. ab30350; dilution 1:200) or stained with their isotype controls IgG (cat. no. ab172730; dilution 1:200; all Abcam) for $30 \mathrm{~min}$ on ice. Following this incubation, cells were washed in Hanks' balanced salt solution (HBSS) supplemented with $2 \%$ FCS and analysed by flow cytometry using FACSDiVa 6.2 software with a (BD Accuri ${ }^{\mathrm{TM}}$ C6 Flow Cytometer (both BD Bioscience).

ELISA. IL6, IL2 and IL8 levels were detected using ELISA. Briefly, cells (LS180, LS180 shPTEN, doxoLS180 and doxoLS180 shPTEN) were seeded at a density of $0.25 \times 10^{6}$ in a 24-well plate and allowed to grow in an incubator at $37^{\circ} \mathrm{C}$ with $5 \% \mathrm{CO}_{2}$ and $95 \%$ humidity for 3 days. The media from the cultured cells was then removed and was analysed for the IL6, IL2 and IL8 levels using an antibody array 5 raybio human cytokine kit according to the manufacturer's protocol. 


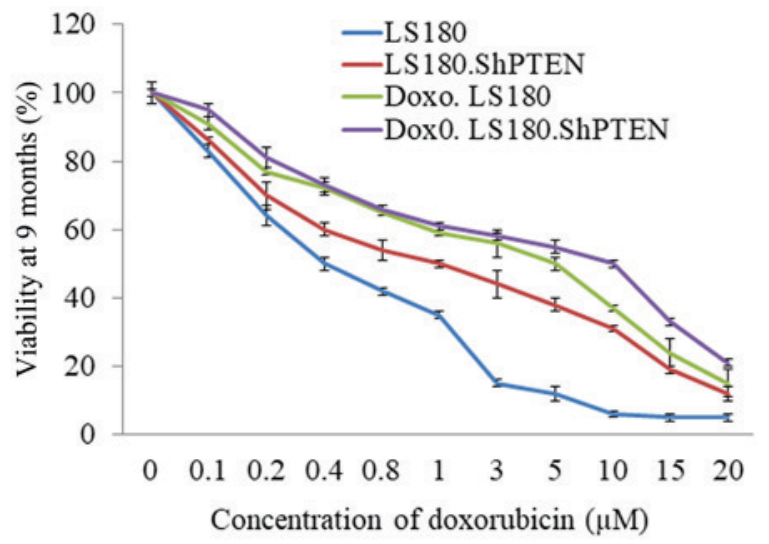

Figure 1. Generation of chemoresistant cells. The colon cancer cell line LS180 was selected and a PTEN knockdown model was generated. Chemoresistance against doxorubicin was applied by treating the cells (LS180 and LS180 shPTEN) with increasing concentrations of doxorubicin ( 0.01 to $20 \mu \mathrm{M}$ ) over a period of 9 months. The $\mathrm{IC}_{50}$ of parental cells was calculated as $0.4 \mu \mathrm{M}$ which was increased to $1 \mu \mathrm{M}$ in LS180 shPTEN cells. However, in resistant clones (doxo LS180 and doxo LS180 shPTEN), the $\mathrm{IC}_{50}$ value increased to 5 and $10 \mu \mathrm{M}$, respectively, confirming the generation of resistance, as determined by MTT assay. The data also demonstrated that PTEN knockdown further enhanced doxorubicin resistance. Data are presented as the mean of three different experiments. $\mathrm{IC}_{50}$, half maximal inhibitory concentration; sh, short hairpin; PTEN, phosphatase and tensin homolog; doxo, doxorubicin-resistant.

$3 D$ sphere formation assay. Using mammocult medium (Stem Cell Technologies, Inc., Vancouver, BC, Canada), cells (LS180, LS180 shPTEN, doxoLS180 and doxoLS180 shPTEN) were seeded in ultra-low attachment plates at a density of $1 \times 10^{5}$ cells/well and allowed to grow for 7 days and were then treated with tocilizumab at a concentration of $1 \mathrm{mg} / \mathrm{l}$ for $48 \mathrm{~h}$. Following treatment, the primary spheres were dissociated by pippeting and single cells were reseeded in ultra-low attachment 6 -well plates at a density of $5 \times 10^{4}$ cells/well in mammary epithelial growth medium (MEGM, Lonza), supplemented with B27 (Invitrogen; Thermo Fisher Scientific, Inc.), $20 \mathrm{ng} / \mathrm{ml}$ bFGF (Sigma-Alrich; Merck KGaA) and $30 \mathrm{ng} / \mathrm{ml} \mathrm{EGF}$ were incubator at $37^{\circ} \mathrm{C}$ with $5 \% \mathrm{CO}_{2}$ and $95 \%$ humidity for 21 days of incubation. A total of 20 fields of view were randomly selected, observed and secondary spheres were counted using a light microscope at a magnification of $\mathrm{x} 30$.

Reverse transcription-quantitative polymerase chain reaction $R T$ - $q P C R$. Primers were obtained from Invitrogen (Thermo Fisher Scientific, Inc.) vimentin forward, 5'-GGCTCAGAT TCAGGGGAACAGC-3' and reverse, 5'-CAGGTTGTG CAGGTTGTTCTA-3'; TWIST forward, 5'-TGTAAAACG ACGGCCAGT-3' and reverse, 5'-CAGGAAACAGCTATG ACC-3'; N-cadherin forward, 5'-CACTGCTCAGGACCC AGAT-3' and reverse, 5'-TAAGCCGAGTGATGGTCC-3'; TGFR forward, 5'-TGTAAAACGACGGCCAGT-3' and reverse, 5'-CAGGAAACAGCTATGACC-3'; E-cadherin forward, 5'-GGGGTACCTGTCTCTCTACAAAAAGGCA-3' and reverse, 5'-GGAAGATCTGGGCTGGAGCGGGCTGGA GT-3'; CLDN3 forward, 5'-CTGCTCTGCTGCTCGTGTCC-3' and reverse, 5'-TTAGACGTAGTCCTTGCGGTCGTAG-3'; EpCAM forward, 5'-CGCAGCTCAGGAAGAATGTG-3' and reverse, 5'-TGAAGTACACTGGCATTGACG-3'; and GAPDH forward, 5'-GGTGTGAACGGATTTGGCCGTATTG-3'; and reverse, 5'-CCGTTGAATTTGCCGTGAGTGGAGT-3' Total RNA was isolated from parental and doxorubicin-resistant cells using the TRIzol reagent (Sigma-Aldrich; Merck KGaA). RNA was purified by using RNeasy mini kit (Qiagen $\mathrm{GmbH}$, Hilden, Germany). RNA was reverse-transcribed into cDNA using M-MLV RT kit (Promega Corporation, Madison, WI, USA). RT-qPCR was performed using a TaqMan universal PCR master mix from Roche (Roche Diagnostics, Basel, Switzerland) with reverse transcription involving denaturation at $94^{\circ} \mathrm{C}$ for $30 \mathrm{sec}$ and annealing and elongation at $72^{\circ} \mathrm{C}$ for 1 min followed by aforementioned primers on an ABI PRISM sequencing detection system (Applied Biosystems; Thermo Fisher Scientific, Inc.) The relative fold change of differential inducible expression of the genes vs. control group was quantified by using the $2^{-\Delta \Delta C q}$ method (27).

Statistical analysis. For statistical analysis graphPad Instat3 software (GraphPad Software Inc., La Jolla, CA, USA) was used. All the experiments were performed three times. The relevant data are expressed as the mean \pm standard deviation (SD). One-way analysis of variance by post-hoc analysis with Tukey's multiple-comparisons test was performed was used to examine differences among multiple groups. $\mathrm{P}<0.05$ was considered to indicate a statistically significant difference.

\section{Results}

Development of a PTEN deficient and chemoresistant cell line. LS180, a colon cancer cell line was selected, and a stable PTEN knockdown model of LS180 was generated. LS180 and LS180 shPTEN cells were treated with increasing concentrations of doxorubicin for a period of over nine months. Resistance developed by doxorubicin was calculated by cell viability. The $\mathrm{IC}_{50}$ of doxorubicin had increased in the resistant cells as compared with their parental cell lines. Furthermore, the knockdown of PTEN also decreased the response of LS180 cells towards doxorubicin (Fig. 1).

Doxorubicin resistance and PTEN knockdown synergize to increase IL6 levels. The IL6 signalling pathway is a crucial cellular pathway and its deregulation has been reported in various types of cancer. Doxorubicin resistance in LS180 cells led to increased level of IL6 which was further elevated in response to shPTEN treatment (Fig. 2A). It was also observed that shPTEN in doxorubicin resistance LS180 leads to an increase in the level of IL2 and IL8 (Fig. 2B). Furthermore, tocilizzumab (an IL6 inhibitor) led to almost complete inhibition of IL6 production in the parental cells as well as doxorubicin-resistant and PTEN knockdown models and this effect was also observed in IL2 and IL8 (Fig. 2A and B). Furthermore, the expression of p-AKT 473 has been increased in doxorubicin resistance LS180 cells that leads to the upregulation of NF-kB as compared with LS180 cells. However, the effect was further elevated in response to shPTEN treatment (Fig. 2C and D).

PTEN knockdown with doxorubicin resistance increases the cancer stem cell population. To investigate the stem properties of different subtype, the present study compared the expression 


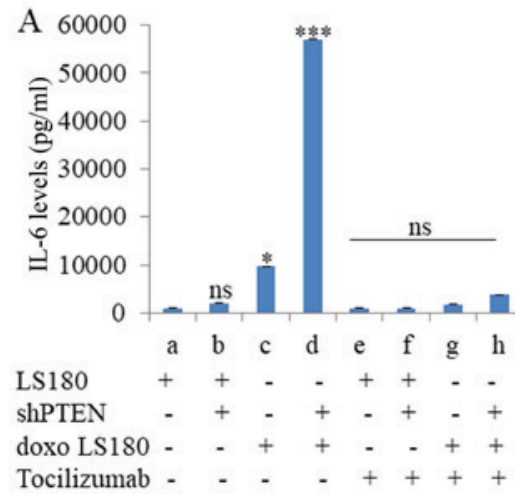

$\mathrm{C}$

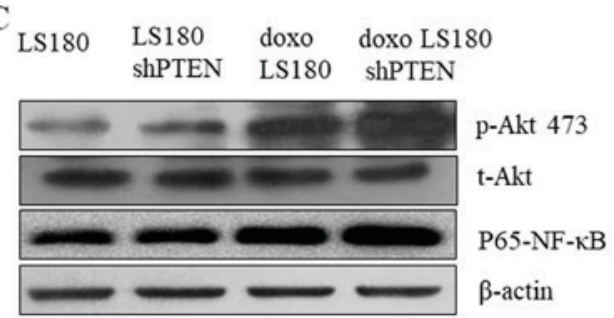

B

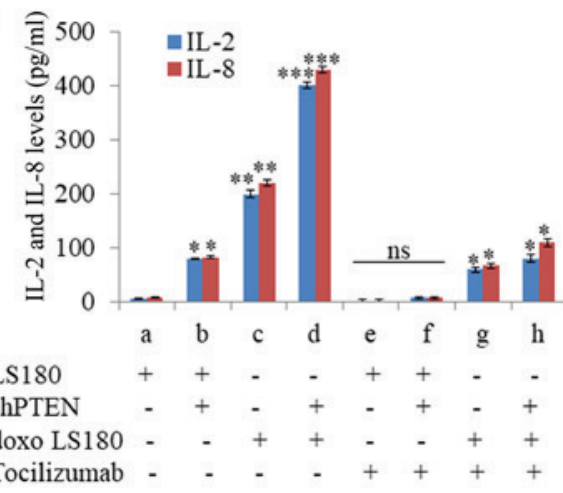

$\mathrm{D}$

Figure 2. Doxorubicin resistance enhances IL6 levels. (A) Doxorubicin resistance enhanced the production of IL6, which was further increased in PTEN knockdown cells as determined by Raybio human cytokine antibody array. However, Tocilizumab antibody at a concentration of $5 \mu \mathrm{g} / \mathrm{ml}$ reduced the IL6 levels by $>90 \%$ in parental cells as well as doxorubicin-resistant models. (B) The levels of IL8 and IL2 due to doxorubicin resistance were reduced by using tocilizumab antibody at a concentration of $5 \mu \mathrm{g} / \mathrm{ml}$. Data presented here are means of three similar experiments. ${ }^{* * *} \mathrm{P}<0.001,{ }^{* *} \mathrm{P}<0.01$, ${ }^{*} \mathrm{P}<0.05$ vs. LS180 (C) Doxorubicin resistance along with knockdown of PTEN led to activation of Akt and P65-NF-kB. (D) Western blot analysis illustrating the knockdown of PTEN in LS180 shPTEN cells. Data are presented the mean of three independent experimental repeats. sh, short hairpin; PTEN, phosphatase and tensin homolog; ns, not significant; doxo, doxorubicin-resistant; NF, nuclear factor; IL, interleukin; R, receptor.

of CD44, CD24 in LS180, LS180 shPTEN, doxoLS180 and doxoLS180 shPTEN cell lines using flow cytometry analysis. As expected doxorubicin resistance led to an increase in the population of these cancer-like stem cells whose numbers were further elevated by knockdown of PTEN by analysing the markers of stem cells such as $\mathrm{CD}_{4} 4^{+} / \mathrm{CD} 24^{-}$(Fig. 3A). Doxorubicin-resistant cells exhibited an increased ability to form mammospheres. PTEN knockdown further increased the formation of mammospheres. Notably, anti-IL6R has decreased the formation of mammospheres in PTEN knockdown doxorubicin resistant LS180 cells (Fig. 3B).

PTEN knockdown with doxorubicin resistance induces epithelial-mesenchymal transition (EMT). Cancer cells undergo reprogramming and change morphologically when treated with chemoresistant drugs over a period of time (28). The results of the present study demonstrated that RT-qPCR analysis of the epithelial to mesenchymal responsive genes including neural (N)-cadherin, vimentin, TWIST and TGFR2 was markedly increased in doxorubicin-resistant cells which was further enhanced by shPTEN doxorubicin-resistant cells as compared with LS180 (Fig. 4A). Furthermore, the expression of E-cadherin, epithelial cell adhesion molecule (EpCAM) and claudin-3 was decreased in both doxorubicin-resistant and shPTEN doxorubicin-resistant cells compared with LS180 cells (Fig. 4A). It was further confirmed by western blotting that there was a increase in the expression of $\mathrm{N}$-cadherin, vimentin, TWIST and TGFR2 in doxorubicin-resistant cells as compared to parental cells (Fig. 4C) whereas an increase in the expression of mesenchymal proteins, including E-cadherin, epithelial cell adhesion molecule (EpCAM) and claudin-3 in the doxorubicin-resistant cells compared with parental cells (Fig. 4D. Taken together, these results demonstrate that doxorubicin leads to resistance in colon cancer and induces EMT-like phenotype which is further elevated by the knockdown of PTEN.

\section{Discussion}

Colon cancer is one of the leading cause of cancer-associated mortalities worldwide, and drug resistance remain a major challenge (29). However, presently there are strategic approaches in pre-clinical trials, including the combination of ATP-binding cassette (ABC) transporters and Epidermal growth factor receptor (EGFR) inhibitors, which are being administered in conjuction with conventional anti-cancer drugs, which have proven effective against drug resistance in colon cancer (30).

In the present study, a PTEN knockdown model of LS180 cells was generated and LS180 and its PTEN knockdown model were subjected to doxorubicin treatment for a period of 8-10 months to generate a drug-resistant cell line against doxorubicin The present study also demonstrated that the fraction of cancer-like stem cells, which have been reported to be responsible for drug resistance, was increased by the knockdown of PTEN (31). Furthermore, it was also observed that the fraction of cancer stem cells was further increased in doxorubicin-resistant cells wherein it was identified that the increased fraction of cancer stem cells was high in PTEN knockdown model of LS180 as compared with doxorubicin-resistant LS180 cells. This may be due to the fact that PTEN acts as a tumor suppressor by negatively acting on the Akt signalling pathway, 
A

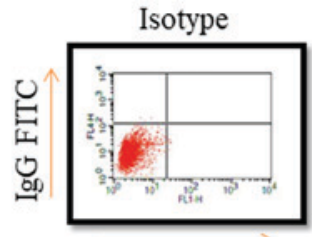

IgG PE

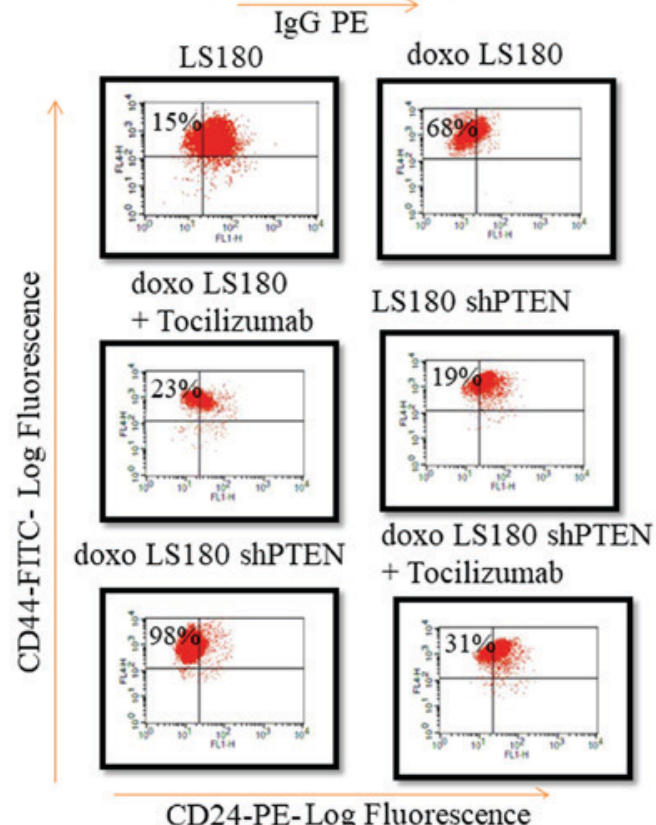

B

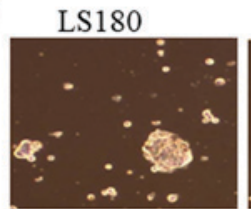

doxo LS180

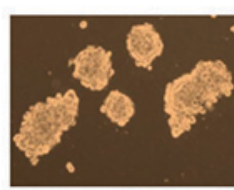

LS180+

Tocilizumab

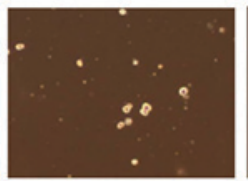

doxo LS180+

Tocilizumab

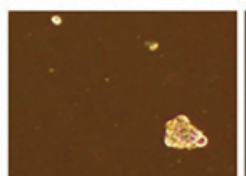

LS180+shPTEN

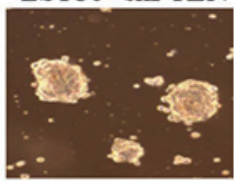

doxo LS180

+ shPTEN

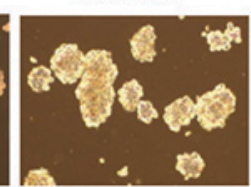

LS180+shPTEN

+ Tocilizumab

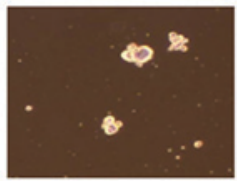

doxo LS180+

Tocilizumab+shPTEN

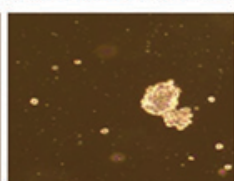

Figure 3. PTEN knockdown along with doxorubicin resistance increases fraction of cancer stem cells. (A) PTEN knockdown increased the fraction of cells expressing CD44 ${ }^{+}$CD24 markers in LS180 shPTEN cells, which was further enhanced by doxorubicin resistance. However, treatment with Tocilizumab antibody led to a decrease in cancer stem cell fraction. (B) PTEN knockdown increased mammosphere formation, which was further enhanced by doxorubicin resistance. However, a marked decrease in mammosphere formation was observed in samples treated with Tocilizumab antibody. Mammospheres were counted using a light microscope at a magnification of x30. sh, short hairpin; PTEN, phosphatase and tensin homolog; doxo, doxorubicin-resistant; FITC, fluorescein isothiocyanate; PE, phycoerythrin; R, receptor.
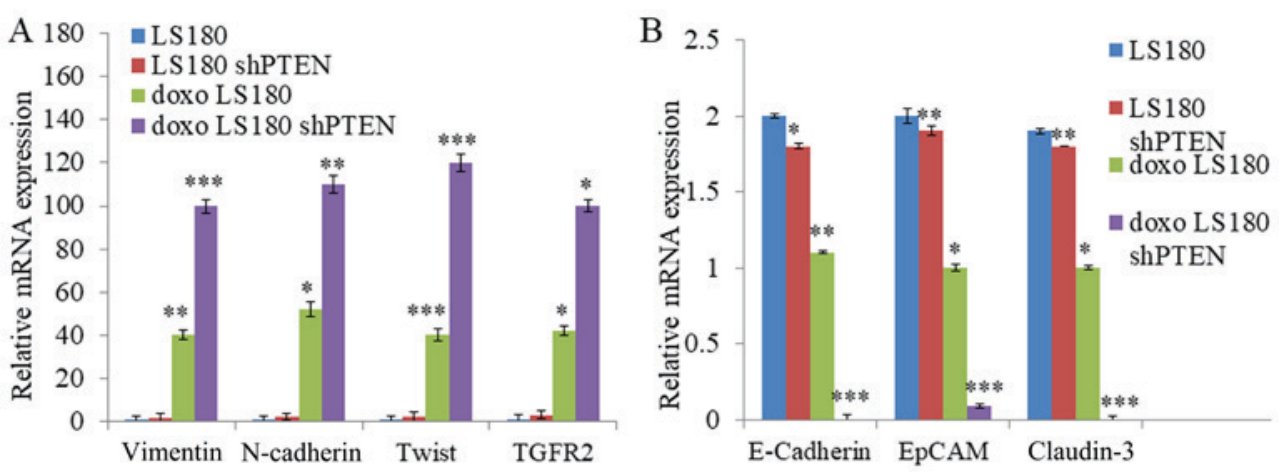

$\mathrm{C}$

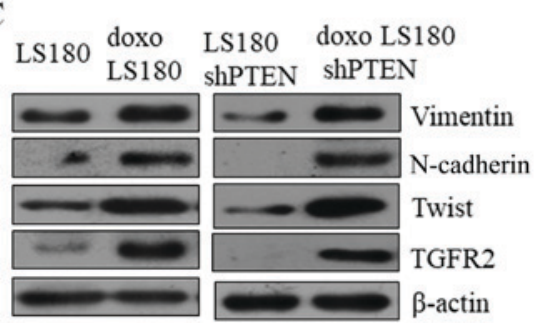

$\mathrm{D}$

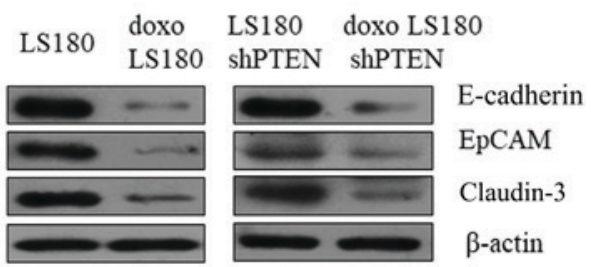

Figure 4. Induction of EMT in LS180 by doxorubicin resistance. (A) Quantification of mesenchymal markers vimentin, N-cadherin, Twist and TGFR2 by RT-qPCR using GAPDH as the normalizing marker. (B) Quantification of epithelial markers E-cadherin, EpCAM and claudin-3 by RT-qPCR using GAPDH as normalizing marker. Data presented here are means of three similar experiments. ${ }^{* * * *} \mathrm{P}<0.001,{ }^{* * *} \mathrm{P}<0.01,{ }^{*} \mathrm{P}<0.05$ vs. LS180. (C) Assessment of EMT phenotype at protein level was analyzed by western blotting in which the expression of epithelial markers was decreased and the expression of mesenchymal markers in doxorubicin-resistant cells was increased. (D) The expression of E-cadherin, EpCAM and claudin-3 decreases in shPTEN doxorubicin-resistant cells compared with parental cells shPTEN LS180 cells. Data are presented the mean of three independent experimental repeats. EMT, epithelial mesenchymal transition; RT-qPCR, reverse transcription-quantitative polymerase chain reaction; sh, short hairpin; PTEN, phosphatase and tensin homolog; doxo, doxorubicin-resistant; TGFR2; transforming growth factor $\beta$ receptor 2; EpCAM, epithelial cell adhesion molecule; E-cadherin, epithelial cadherin; N-cadherin, neural cadherin. 
which is a pathway that has been identified to be deregulated in the majority types of cancer (31). PTEN mutations may contribute to the failure of chemotherapy and therefore drug resistance (32). The results of the present study demonstrated that there was an increase in the levels of IL6, IL2 and IL8. Knockdown of PTEN led to an increase in the levels of IL6 which were further increased in the doxorubicin-resistant cells. These results indicated that a positive feedback loop is generated whereby IL6 increased expression of the NF- $\kappa B$ pathway, which further enhances the production of IL6, thus linking inflammation to the malignant transformation of tumours. Doxorubicin resistance also induced epithelial to mesenchymal transition in the colon cancer cell line LS180 and its PTEN knockdown model as it was demonstrated by the decrease in the expression of epithelial marker E-cadherin in resistant cells as compared with parental cells. Additionally, notable changes were shown in the expression of mesenchymal markers, including vimentin and $\mathrm{N}$-cadherin in the drug-resistant cells compared with parental cells at the mRNA and protein level as was examined by RT-qPCR and western blotting.

To conclude, investigating the underlying mechanisms responsible for drug resistance is of great clinical significance. The present study was able to demonstrate that doxorubicin resistance may lead to the increased expression of IL6 signalling pathway which was further activated by knockdown of PTEN, and this effect may be reversed using an anti-IL6R antibody. Additionally, the increase in IL6 levels due to doxorubicin resistance led to the expansion of cancer stem cells and induced EMT.

\section{Acknowledgements}

Not applicable.

\section{Funding}

Financial assistance for the present study was provided the National Natural Science Foundation of China (grant no. 2015DB000475).

\section{Availability of data and materials}

In the present study the datasets generated and analyzed are included in this published article.

\section{Authors' contributions}

The experiments was performed and analyzed by XYL and XFL. Study design and manuscript preparation by HBW and JZ.

\section{Ethics approval and consent to participate}

The present study has been approved by the Ethics Committee of Xiangyang Central Hospital and written informed consent was obtained from all participants.

\section{Patient consent for publication}

The present study participants provided consent for the data and any associated images to be published.

\section{Competing interests}

The authors declare that they have no competing interests.

\section{References}

1. Gandomani HS, Yousefi SM, Aghajani M, Mohammadian-Hafs hejani A, Tarazoj AA, Pouyesh V and Salehiniya H: Colorectal cancer in the world: Incidence, mortality and risk factors. Biomed Res Therapy 4: 10, 2017.

2. Haggar FA and Boushey RP: Colorectal cancer epidemiology: Incidence, mortality, survival, and risk factors. Clin Colon Rectal Surg 22: 191-197, 2009.

3. Mishra J, Dromund J, Quazi SH, Karanki SS, Shaw JJ, Chen B and Kumar N: Prospective of colon cancer treatments and scope for combinatorial approach to enhanced cancer cell apoptosis. Crit Rev Oncol Hematol 86: 232-250, 2013.

4. Wáng YX, De Baere T, Idée JM and Ballet S: Transcatheter embolization therapy in liver cancer: An update of clinical evidences. Chin J Cancer Res 27: 96-121, 2015.

5. Blakely T, Collinson L Kvizhinadze G, Nair N, Foster R, Dennett E and Sarfati D: Cancer care coordinators in stage III colon cancer: A cost-utility analysis. BMC Health Serv Res 15: 306, 2015.

6. Keating NL, Landrum MB, Lamont EB, Bozeman SR, Krasnow SH, Shulman LN, Brown JR, Earle CC, Oh WK, Rabin M and McNeil BJ: Quality of care for older patients with cancer in the Veterans health administration versus the private sector: A cohort study. Ann Intern Med 154: 727-736, 2011.

7. Lam TJ, Meurs-Szojda MM, Gundlach L, Belien JA, Meijer GA, Mulder CJ and Felt-Bersma RJ: There is no increased risk for colorectal cancer and adenomas in patients with diverticulitis: A retrospective longitudinal study. Colorectal Dis 12: 1122-1126, 2010.

8. Suraj R, Radhamani S, Meehan-Andrews T and Bradley C: Role of a novel benzoxazine derivative in the chemosensitization of colon cancer. Apoptosis 22: 988-1000, 2017.

9. Kadioglu O, Law BYK, Mok SWF, Xu SW, Efferth T and Wong VKW: Mode of action analyses of neferine, a bisbenzylisoquinoline alkaloid of lotus (Nelumbo nucifera) against multidrug-resistant tumor cells. Front Pharmacol 8: 238, 2017.

10. Kang XJ, Wang HY, Peng HG, Chen BF, Zhang WY, Wu AH, $\mathrm{Xu} \mathrm{Q}$ and Huang YZ: Codelivery of dihydroartemisinin and doxorubicin in mannosylated liposomes for drug-resistant colon cancer therapy. Acta Pharmacol Sin 38: 885-896, 2017.

11. Xu J, Zhang S, Wang R, Wu X, Zeng L and Fu Z: Knockdown of PRDX2 sensitizes colon cancer cells to 5-FU by suppressing the PI3K/AKT signaling pathway. Biosci Rep 37: BSR20160447, 2017.

12. Nedaeinia R, Avan A, Ahmadian M, Nia SN, Ranjbar M, Sharifi M, Goli M, Piroozmand A, Nourmohammadi E, Manian $\mathrm{M}$, et al: Current status and perspectives regarding LNA-Anti-miR oligonucleotides and microRNA miR-21 inhibitors as a potential therapeutic option in treatment of colorectal cancer. J Cell Biochem 118: 4129-4140, 2017.

13. Sambi M, Haq S, Samuel V, Qorri B, Haxho F, Hill K, Harless W and Szewczuk MR: Alternative therapies for metastatic breast cancer: Multimodal approach targeting tumor cell heterogeneity. Breast Cancer (Dove Med Press) 9: 85-93, 2017.

14. Schoumacher M and Burbridge M: Key roles of AXL and MER receptor tyrosine kinases in resistance to multiple anticancer therapies. Curr Oncol Rep 19: 19, 2017.

15. Zheng D, Wu W, Dong N, Jiang X, Xu J, Zhan X, Zhang Z and Hu Z: Mxd1 mediates hypoxia-induced cisplatin resistance in osteosarcoma cells by repression of the PTEN tumor suppressor gene. Mol Carcinog 56: 2234-2244, 2017.

16. El Ouar I, Braicu C, Naimi D, Irimie A and Berindan-Neagoe I: Effect of Helix aspersa extract on TNF $\alpha, N F-\kappa B$ and some tumor suppressor genes in breast cancer cell line Hs578T. Pharmacogn Mag 13: 281-285, 2017.

17. Mantamadiotis T: Towards targeting PI3K-dependent regulation of gene expression in brain cancer. Cancers (Basel) 9: E60, 2017.

18. Ban HS, Kim BK, Kim HM, Harmalkar D, Nam M, Park SK, Lee K, Park JT, Kim I, et al: The novel hypoxia-inducible factor-1 $\alpha$ inhibitor IDF-11774 regulates cancer metabolism, thereby suppressing tumor growth. Cell Death Dis 8: e2843, 2017. 
19. Tesio M, Trinquand A, Ballerini P, Hypolite G, Lhermitte L, Petit A, Ifrah N, Baruchel A, Dombret H, Macintyre E and Asnafi V: Age-related clinical and biological features of PTEN abnormalities in T-cell acute lymphoblastic leukaemia. Leukemia 31: 2594-2600, 2017.

20. Li Z, Dong X, Wang Z, Liu W, Deng N, Ding Y, Tang L, Hla T, Zeng R, Li L and Wu D: Regulation of PTEN by Rho small GTPases. Nat Cell Biol 7: 399-404, 2005.

21. Solari F, Bourbon-Piffaut A, Masse I, Payrastre B, Chan AM and Billaud M: The human tumour suppressor PTEN regulates longevity and dauer formation in Caenorhabditis elegans. Oncogene 24: 20-27, 2005.

22. Bar N and Dikstein R: miR-22 forms a regulatory loop in PTEN/AKT pathway and modulates signaling kinetics. PLoS One 5: e10859, 2010.

23. Chalhoub $\mathrm{N}$ and Baker SJ: PTEN and the PI3-Kinase pathway in cancer. Annu Rev Pathol 4: 127-150, 2009.

24. Keniry M and Parsons R: The role of PTEN signaling perturbations in cancer and in targeted therapy. Oncogene 27: 5477-5485, 2008.

25. Korkaya H, Kim GI, Davis A, Malik F, Henry NL, Ithimakin S, Quraishi AA, Tawakkol N, D'Angelo R, Paulson AK, et al: Activation of an IL-6 inflammatory loop mediates trastuzumab resistance in HER2+ breast cancers by expanding the cancer stem cell population. Mol Cell 47: 570-584, 2012.

26. Zarogoulidis P, Yarmus L, Darwiche K, Walter R, Huang H, Li Z, Zaric B, Tsakiridis K and Zarogoulidis K: Interleukin-6 cytokine: A multifunctional glycoprotein for cancer. Immunome Res 9: 16535, 2013.
27. Livak KJ and Schmittgen TD: Analysis of relative gene expression data using real-time quantitative PCR and the 2(-Delta Delta C(T)) method. Methods 25: 402-408, 2001

28. El-Badawy A, Ghoneim MA, Gabr MM, Salah RA, Mohamed IK, Amer M and El-Badri N: Cancer cell-soluble factors reprogram mesenchymal stromal cells to slow cycling, chemoresistant cells with a more stem-like state. Stem Cell Res Ther 8: 254, 2017.

29. Hu T, Li Z, Gao CY and Cho CH: Mechanisms of drug resistance in colon cancer and its therapeutic strategies. World J Gastroenterol 22: 6876-6889, 2016.

30. Hammond WA, Swaika A and Mody K: Pharmacologic resistance in colorectal cancer: A review. Ther Adv Med Oncol 8: 57-84, 2016.

31. Pu H, Zheng Q, Li H, Wu M, An J, Gui X, Li T and Lu D: CUDR promotes liver cancer stem cell growth through upregulating TERT and C-Myc. Oncotarget 6: 40775-40798, 2015.

32. Phadngam S, Castiglioni A, Ferraresi A, Morani F, Follo C and Isidoro C: PTEN dephosphorylates AKT to prevent the expression of GLUT1 on plasmamembrane and to limit glucose consumption in cancer cells. Oncotarget 7: 84999-85020, 2016.

This work is licensed under a Creative Commons Attribution-NonCommercial-NoDerivatives 4.0 International (CC BY-NC-ND 4.0) License. 